\title{
Pointing towards colonoscopy: sharp foreign body removal via colonoscopy
}

\author{
Melissa Hershman, Steven Shamah, Prashant Mudireddy, Michael Glick \\ Lenox Hill Hospital, N.Y., USA
}

\begin{abstract}
Removal of sharp foreign bodies via upper endoscopy is common; however, management in the setting of distal migration is not well-documented. We report two cases of objects beyond the ligament of Treitz, including successful extraction of a $4.4 \mathrm{~cm}$ sewing pin from the cecum using hot biopsy forceps with a protector hood to shield colonic mucosa, and in a separate case, a $3.4 \mathrm{~cm}$ glass shard from the ascending colon using a Roth Net retriever. We demonstrate that monitoring with serial radiographs and examination may allow for supervised passage of sharp objects into the colon, where removal can be performed safely via colonoscopy.
\end{abstract}

Keywords Sharp objects, foreign body, colonoscopy

Ann Gastroenterol 2017; 29 (4): 1-3

\section{Introduction}

Ingestion of foreign bodies is common and well-documented. Pre-endoscopic historical studies have shown that a majority of foreign bodies pass spontaneously, with mortality rates under $0.1 \%$ [1]. Sharp objects, including chicken and fish bones, needles, toothpicks and dental implants, raise unique concerns, given that bowel perforation rates up to $35 \%$ have been reported, typically at the ileocecal valve, and less commonly at colonic flexures and the rectosigmoid junction [1,2]. Patients are also at risk for small bowel obstruction, particularly with objects larger than $6 \mathrm{~cm}$ [2]. The safety and efficacy of foreign body removal via upper endoscopy is well-established, without increased morbidity [3]. For these reasons, the American Society for Gastrointestinal Endoscopy (ASGE) recommends emergent endoscopy for sharp-pointed objects in the esophagus and urgent endoscopy for objects located in the stomach or duodenum [4].

Once foreign bodies have passed beyond the reach of the endoscope, management becomes less objective, with limited literature to evaluate the safety of sharp object removal via colonoscopy. We report two cases in which patients were successfully managed via a colonoscopic approach.

Department of Medicine, Internal Medicine Resident, Lenox Hill Hospital, N.Y., USA

\section{Conflict of Interest: None}

Correspondence to: Melissa Hershman, M.D., R.N., Lenox Hill Hospital, Department of Medicine, Internal Medicine Resident, 100 E. $77^{\text {th }}$ Street New York, N.Y. 10075, USA,

e-mail: mhershman@northwell.edu

Received 07 October 2016; accepted 29 November 2016; published online 22 December 2016

DOI: https://doi.org/10.20524/aog.2016.0116

\section{Case 1}

A 25-year-old female seamstress presented after accidental ingestion of a $4.4 \mathrm{~cm}$ pin. Physical examination, comprehensive metabolic panel, and complete blood count were unremarkable. Plain film radiographs demonstrated a radiopaque $4.4 \mathrm{~cm}$ needlelike structure over the distal gastric body without pneumoperitoneum. Computed tomography (CT) imaging without contrast $2 \mathrm{~h}$ later showed the foreign body in the distal duodenum. As the needle was distal to the ligament of Treitz (Fig. 1), the decision was made to follow the patient conservatively per ASGE guidelines. After serial plain film imaging found no interval migration from the right lower quadrant by day 3 , she was given oral bowel preparation for colonoscopy. On colonoscopy, the pin was freely mobile in the cecum and not impacted in the colon wall. Using hot biopsy forceps, the sharp end of the pin was secured and the scope carefully withdrawn with the hood pulled forward over the entire needle (Fig. 2). At about $40 \mathrm{~cm}$, the pin jarred loose from the forceps. Because of the poor visualization due to the hood being pulled forward, the scope was withdrawn completely and the hood repositioned. The scope was reinserted and the same technique was used to secure the sharp aspect with forceps before pulling the entire pin into the hood. Once the foreign body was removed, the scope was reinserted to the sigmoid colon, revealing intact mucosa without perforation or injury. The patient was observed for $24 \mathrm{~h}$ and discharged without adverse events.

\section{Case 2}

A 23-year-old female reported to the emergency department after accidently drinking from a broken glass. Physical exam findings and routine laboratory studies were benign. An abdominal $\mathrm{X}$-ray demonstrated a proximal $3.4 \mathrm{~cm}$ linear opaque density in the 
left abdomen (Fig. 3). Endoscopy was performed, but the object could not be visualized and was thought to be beyond the reach of the endoscope. Abdominal X-rays were performed 2-3 times daily, localizing the object to the cecum on hospital day 2 without any advancement by day 4 . During colonoscopy, the glass was identified in the mid-ascending colon and retrieved using a Roth Net retriever (Fig. 4). The scope was completely withdrawn after retroflexion with careful examination of the colonic mucosa. The follow-up X-ray showed absence of the object and no perforation. The patient was discharged after $24 \mathrm{~h}$ of observation.

\section{Discussion}

Practitioners must maintain a high index of clinical suspicion when faced with possible sharp object ingestion. Personal risk

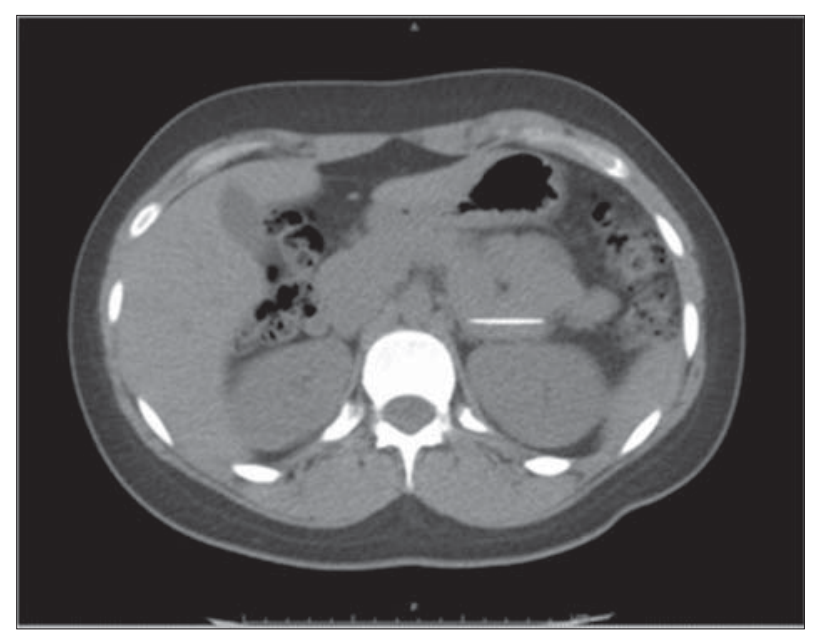

Figure 1 Non-contrast computed tomography showing a radiopaque foreign body compatible with the patient's history of a swallowed needle, located in the distal portion of the third segment of the duodenum/ligament of Treitz

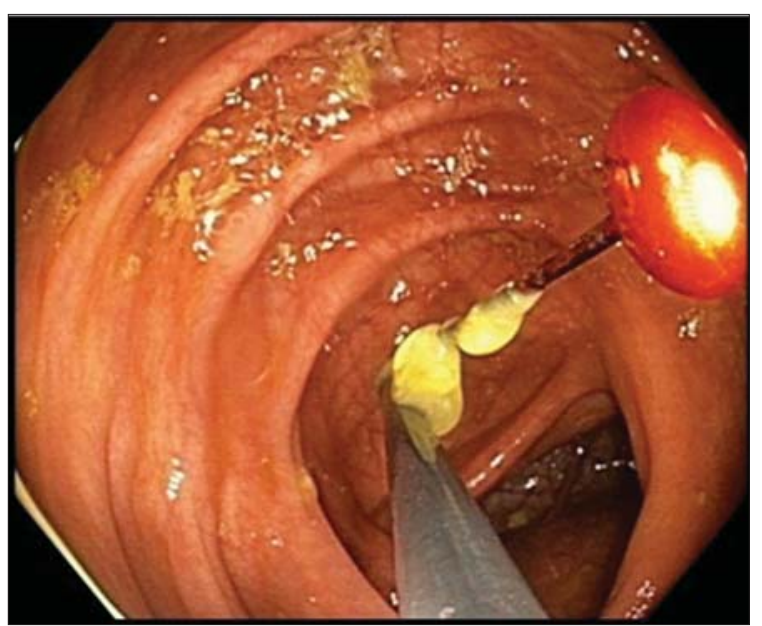

Figure 2 Extraction of the foreign body from the cecum using endoscopic forceps to grasp the sharp pin tip factors in adult populations include developmental delay, alcohol intoxication, psychiatric illness and incarceration [5]. Even in the absence of radiographic evidence, an endoscopic study is still advised and should be performed on an urgent or emergent basis [4].

When sharp objects have migrated beyond the ligament of Treitz, as in the cases presented here, the course of action is more ambiguous. Biplane radiographs should be followed daily to trace passage [2]; however, objects of wood, glass, and plastic composition may not be readily visible. CT imaging, particularly with three-dimensional reconstruction, may improve visualization [6].

Conventional practice advocates for surgical intervention if the object has not passed after 3-4 days [4,7]. A retrospective series found that surgery was required in $4.8 \%$ of 542 cases,

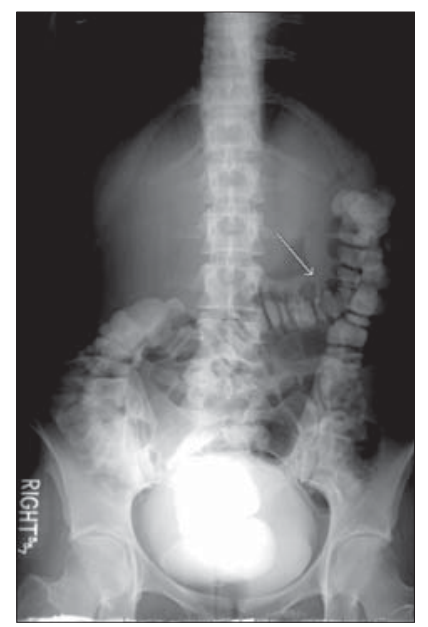

Figure 3 Upright abdominal radiograph showing a proximal 3.4-cm linear opaque density (arrow) overlying the left abdomen with nonspecific bowel gas pattern. Visualization of objects of certain composition, such as the glass pictured here, requires astute observation and clinical correlation

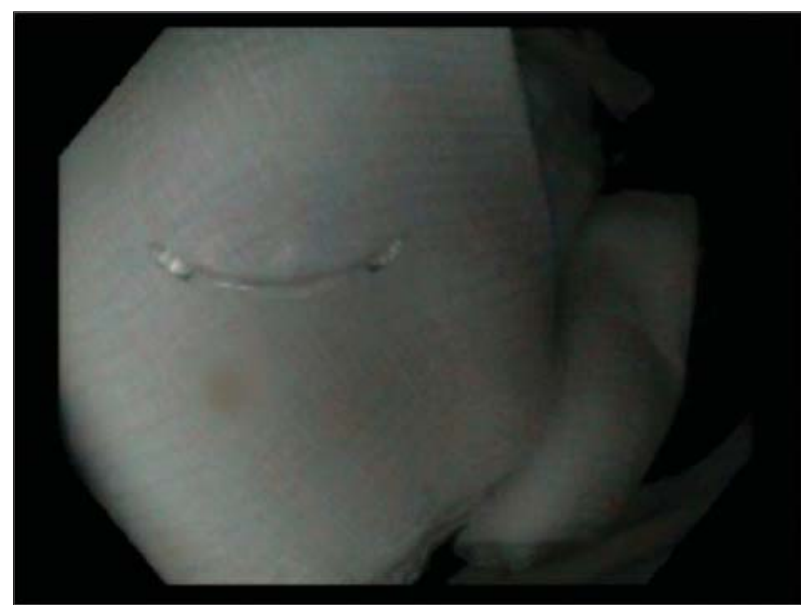

Figure 4 Narrow-band imaging utilized during colonoscopy to improve the visualization of the glass object in the mid-ascending colon 
$15.4 \%$ of which were for sharp object perforation and $53.8 \%$ for ileocecal impaction [3]. To date, there is a paucity of evidence to substantiate colonoscopy in patients who have no peritoneal symptoms. Available examples include a $3-\mathrm{cm}$ dental needle in the cecum using endoscopic forceps [8]. More invasive extractions are exemplified by a toothpick impacting the rectosigmoid wall, removed by polypectomy snare [9]; a chicken bone perforating sigmoid mucosa, detached by endoscopic snare under laparoscopic evaluation [6]; and a mucosal bridge entrapping a plastic twist-tie, abolished by injection sclerotherapy, electrocautery and rat-tooth forceps [10]. Analogous to an upper endoscopic approach, devices include retrieval forceps or baskets, as in our examples, or polypectomy snares [4,7]. While during upper endoscopy it is preferable to trail the pointed end during withdrawal, colonoscopic retrieval differs in this respect. We believe that the sharp end of the object should be firmly grasped and covered by forceps, then drawn toward the scope, with the bowel lumen maintained at the center of the visual field to avoid mucosal injury or perforation at the splenic and hepatic flexures. Retracting the object completely into the overtube or protector hood before cautiously withdrawing the entire scope may decrease the chance of mucosal injury during extraction. Once the object is removed, a second-look colonoscopy or follow-up X-ray may reassess for complications, including perforation.

In conclusion, sharp object ingestion poses a unique set of risks, including perforation and bowel obstruction. While prior practice standards advocated for surgical removal when movement had ceased after three days, we recommend consideration of a conservative approach to objects beyond the pylorus by tracking migration on serial X-ray or CT imaging, frequent abdominal examinations, and routine laboratory studies. In the absence of peritoneal signs or clinical instability, our cases, in concordance with the available literature, suggest that a minimally invasive approach via colonoscopy can be a safe, efficient means of removing sharp objects to reduce distal perforation risk and the need for surgery.

\section{References}

1. Carp L. Foreign bodies in the intestine. Ann Surg 1927;85:575-591.

2. Eisen GM, Baron TH, Dominitz JA, et al; American Society for Gastrointestinal Endoscopy. Guideline for the management of ingested foreign bodies. Gastrointest Endosc 2002;55:802-806.

3. Velitchkov NG, Grigorov GI, Losanoff JE, Kjossev KT. Ingested foreign bodies of the gastrointestinal tract: retrospective analysis of 542 cases. World J Surg 1996;20:1001-1005.

4. Ikenberry SO, Jue TL, Anderson MA, et al; ASGE Standards of Practice Committee. Management of ingested foreign bodies and food impactions. Gastrointest Endosc 2011;73:1085-1091.

5. Palta R, Sahota A, Bemarki A, Salama P, Simpson N, Laine L. Foreign-body ingestion: characteristics and outcomes in a lower socioeconomic population with predominantly intentional ingestion. Gastrointest Endosc 2009;69:426-433.

6. Chang KJ, Schechter S, Vrees M, Lidofsky S. Ingested foreign body in the sigmoid colon: detection and localization by CT colonography. Med Health R I 2012;95:47-48.

7. Tang SJ. Endoscopic management of foreign bodies in the gastrointestinal tract. VideoGIE 2012:1:35-38.

8. Kang GC, Madhukumar P. Successful management of an iatrogenically-ingested sharp foreign body. Ann Acad Med Singapore 2008;37:980-981.

9. Zezos P, Oikonomou A, Souftas V, Gkotsis D, Pitiakoudis M, Kouklakis G. Endoscopic removal of a toothpick perforating the sigmoid colon and causing chronic abdominal pain: a case report. Cases J 2009;2:8469.

10. Ramirez FC, Zierer ST, Mills MR, Sanowski RA. Endoscopic removal of an entrapped foreign body from the sigmoid colon using a needle knife. Gastrointest Endosc 1996;44:84-85. 